\title{
Kaon Femtoscopy in $\sqrt{s_{N N}}=200 \mathrm{GeV}$ Central Au+Au Collisions at RHIC-STAR
}

\author{
Paul Chung*t \\ Nuclear Physics Institute, Academy of Sciences of the Czech Republic, Prague, Czech Republic \\ E-mail: pchung_anami@yahoo.com
}

\section{for the STAR Collaboration}

\begin{abstract}
Three-dimensional source functions for mid-rapidity kaon pairs have been extracted for multiple transverse momentum bins from central $\mathrm{Au}+\mathrm{Au}$ collisions data at $\sqrt{s_{N N}}=200 \mathrm{GeV}$ by the STAR experiment at RHIC. The kaon source functions are essentially Gaussian in shape. The extracted Gaussian source radii are in good quantitative agreement with transport calculations from the hydrokinetic model while exhibiting a marked deviation from predictions of the Buda-Lund hydrodynamic model in the longitudinal direction, thereby invalidating the transverse mass scaling feature inherent to that model.
\end{abstract}

The Seventh Workshop on Particle Correlations and Femtoscopy

September 20 - 242011

University of Tokyo, Japan

\footnotetext{
${ }^{*}$ Speaker.

${ }^{\dagger}$ This work was supported by grant LC07048 of MSMT of the Czech Republic.
} 


\section{Introduction}

Three-dimensional source extraction probes the space-time characteristics of the particle emitting fireball created in ultra-relativistic heavy ion collisions [1,2,3]. These femtoscopic techniques exploit the correctly measured two-particle correlation function to yield a two-particle source function with high integrity. Model comparison of the correlation or source function then offers a window into the dynamical processes driving the evolution of the fireball. Such techniques have been successfully applied on pion and kaon data at low transverse momentum at RHIC energies $[4,5]$. Contrary to pions, the extracted kaon source function at low transverse momentum was found to be essentially Gaussian in shape [6]. However, due to the scarcity of sufficiently high quality kaon data, differences in kaon predictions arising from various hydrodynamic scenarios of the fireball expansion could not be unambiguously ascertained. In particular, the transverse mass scaling of Gaussian source radii, which is a natural prediction of the Buda-Lund model [7] of the hydrodynamic expansion but which holds true only approximately in the microscopic hydrokinetic transport model [8], was never fully verified. By presenting new results obtained from application of state-of-the-art femtoscopic techniques at multiple transverse momenta for kaon data collected by the STAR experiment from Au+Au collisions at $\sqrt{s_{N N}}=200 \mathrm{GeV}$, we confront the models with high statistics data and hence aim to resolve the outstanding issue relating to the transverse mass scaling of Gaussian source radii.

\section{Experimental Setup and Data Analysis}

The data presented here were taken by the STAR Collaboration during the year-2004 run. The colliding beams of gold nuclei $\left(\sqrt{s_{N N}}=200 \mathrm{GeV}\right)$ were provided by the RHIC accelerator. Charged tracks were detected in the STAR Time Projection Chamber (TPC) [9], covering \pm 1.1 units of pseudo-rapidity, surrounded by a solenoidal magnet providing a nearly uniform magnetic field of 0.5 Tesla along the beam direction. The TPC provides tracking information for track reconstruction and particle identification by means of energy loss in the TPC gas.

The 3D correlation function, $\mathrm{C}(\mathbf{q})$, was calculated as the ratio of foreground to background distributions in relative momentum $\mathbf{q}$ for $K^{+} K^{+}$and $K^{-} K^{-}$pairs. Here, $\mathbf{q}=\frac{\left(\mathbf{p}_{1}-\mathbf{p}_{2}\right)}{2}$ is half of the relative momentum between the two particles in the pair C.M. system (PCMS) frame. The foreground distribution was obtained using pairs of particles from the same event and the background was obtained by pairing particles from different events. Track merging and splitting effects were removed by appropriate cuts in the relevant coordinate space on both the foreground and background distributions [10]. There was no significant effect on the correlation function due to the momentum resolution of about 3\% for the transverse momentum range under consideration.

In the Cartesian surface-spherical harmonic decomposition technique [3], the 3D correlation function is expressed as

$$
C(\mathbf{q})-1=R(\mathbf{q})=\sum_{l} \sum_{\alpha_{1} \ldots \alpha_{l}} R_{\alpha_{1} \ldots \alpha_{l}}^{l}(q) A_{\alpha_{1} \ldots \alpha_{l}}^{l}\left(\Omega_{\mathbf{q}}\right)
$$

where $l=0,1,2, \ldots, \alpha_{i}=x, y$ or $z, A_{\alpha_{1} \ldots \alpha_{l}}^{l}\left(\Omega_{\mathbf{q}}\right)$ are Cartesian harmonic basis elements $\left(\Omega_{\mathbf{q}}\right.$ is solid 
angle in $\mathbf{q}$ space) and $R_{\alpha_{1} \ldots \alpha_{l}}^{l}(q)$ are Cartesian correlation moments given by

$$
R_{\alpha_{1} \ldots \alpha_{l}}^{l}(q)=\frac{(2 l+1) ! !}{l !} \int \frac{d \Omega_{\mathbf{q}}}{4 \pi} A_{\alpha_{1} \ldots \alpha_{l}}^{l}\left(\Omega_{\mathbf{q}}\right) R(\mathbf{q}),
$$

where $q$ is the modulus of $\mathbf{q}$. The coordinate axes are oriented so that $z$ is parallel to the beam (longitudinal) direction, $x$ points in the direction of the total momentum of the pair in the Locally Co-Moving System (LCMS) frame (outward) and $y$ is perpendicular to $x$ and $z$ (sideward direction).

The correlation moments, for each order $l$, can be calculated from the measured 3D correlation function using Eq. (2.2). In this analysis, Eq. (2.1) is truncated at $l=4$ and expressed in terms of independent moments only. Up to order 4 , there are 6 independent moments: $R^{0}, R_{x 2}^{2}, R_{y 2}^{2}, R_{x 4}^{4}$, $R_{y 4}^{4}$ and $R_{x 2 y 2}^{4}$, where $R_{x 2}^{2}$ is shorthand for $R_{x x}^{2}$ etc. These independent moments were extracted as a function of $q$, by fitting the truncated series to the measured 3D correlation function with the moments as the parameters of the fit. Higher order moments were found to be negligible.

The 3D source function $S(\mathbf{r})$ can be extracted by directly fitting the 3D correlation function with an assumed functional form for $S(\mathbf{r})$. Since the 3D correlation function is represented by the 6 independent correlation moments, the fitting procedure corresponds to a simultaneous fit of the 6 independent correlation moments with the assumed functional form via the 3D Koonin equation Eq. (2.3),

$$
C(\mathbf{q})-1=R(\mathbf{q})=\int d \mathbf{r} K(\mathbf{q}, \mathbf{r}) S(\mathbf{r})
$$

where $K(\mathbf{q}, \mathbf{r})$ is a six-dimensional kernel in which the effects of quantum statistics (Bose-Einstein symmetrisation of like-boson pair wavefunction) and Coulomb interaction (the sources of the observed correlations) are incorporated. In this analysis, the assumed functional form of $S(\mathbf{r})$ used is that of a 3D Gaussian or ellipsoid given by Eq. (2.4),

$$
S^{G}\left(r_{x}, r_{y}, r_{z}\right)=\frac{\lambda}{(2 \sqrt{\pi})^{3} R_{x} R_{y} R_{z}} \exp \left[-\left(\frac{r_{x}^{2}}{4 R_{x}^{2}}+\frac{r_{y}^{2}}{4 R_{y}^{2}}+\frac{r_{z}^{2}}{4 R_{z}^{2}}\right)\right],
$$

where $\lambda, R_{x}, R_{y}$ and $R_{z}$ are fit parameters.

\section{Results}

Figure 1 shows the correlation moments $R_{\alpha_{1} \ldots \alpha_{l}}^{l}$ up to order $l=4$ (open circles) for mid-rapidity $(|y|<0.5)$, low transverse momentum $\left(0.2<k_{T}<0.36 \mathrm{GeV} / \mathrm{c}\right)$ kaon pairs produced in central $(0-30 \%) \mathrm{Au}+\mathrm{Au}$ collisions at $\sqrt{S_{N N}}=200 \mathrm{GeV}$. In panel (a), $R^{0}(q)$ is shown along with the onedimensional correlation function $R(q)=C(q)-1$ (solid circles); both represent angle-averaged correlation functions, but $R^{0}(q)$ is obtained from the three-dimensional correlation function via Eq. (2.2) while $R(q)$ is evaluated directly from the one-dimensional correlation function.

The very good agreement between $R^{0}(q)$ and $R(q)$ underlines the absence of any significant angular acceptance issues and attests to the reliability of the moment extraction technique. Figures 1(b)-(f) show that contributions decrease with increasing $l$ in each direction and are essentially 0 for $l=4$. This justifies truncating Eq. (2.1) at $l=4$. A 4-parameter ellipsoid (three-dimensional 


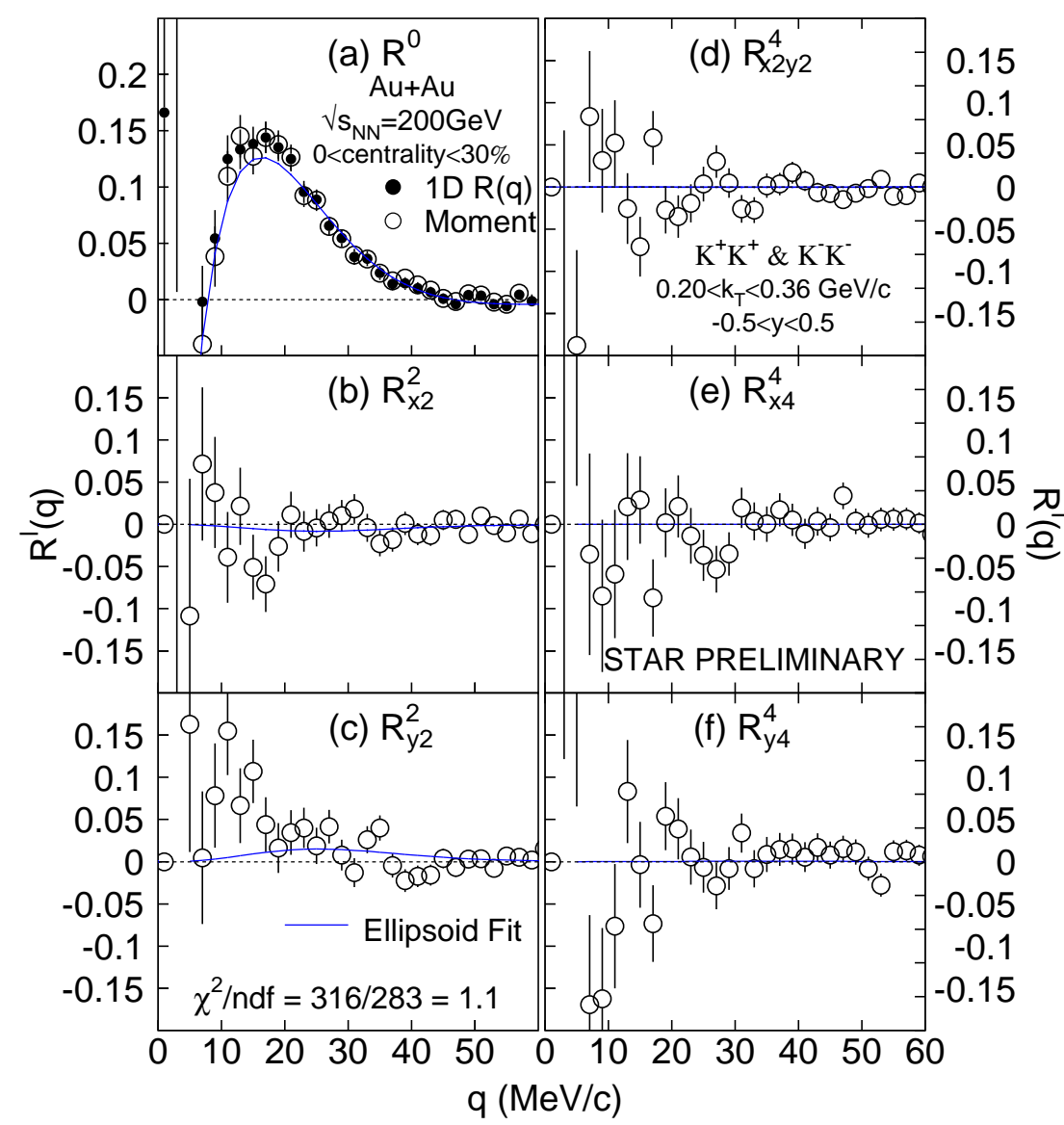

Figure 1: Experimental correlation moments $R^{l}(q)$ for orders $l=0,2,4$ for mid-rapidity kaon pairs $\left(0.20<k_{T}<0.36 \mathrm{GeV} / \mathrm{c}\right)$ from central $(0-30 \%) A u+A u$ collisions at $\sqrt{s_{N N}}=200 \mathrm{GeV}$. Panel (a) also shows a comparison between $R^{0}(q)$ and $1 D$ correlation function $R(q)$. Systematic errors are negligible compared to statistical errors. The solid curve represents the ellipsoid fit to the data.

Gaussian) fit, using MINUIT minimization, gives a reasonable result $\left(\chi^{2} / \mathrm{ndf}=1.1\right)$. The solid curve in Fig. 1 shows the result of the fit to the independent correlation moments.

Figure 2 shows the corresponding correlation moments $R_{\alpha_{1} \ldots \alpha_{l}}^{l}$ up to order $l=4$ (open circles) for mid-rapidity $(|y|<0.5)$ kaon pairs at higher pair transverse momentum $\left(0.36<k_{T}<\right.$ $0.48 \mathrm{GeV} / \mathrm{c})$ produced in these same central $(0-30 \%) \mathrm{Au}+\mathrm{Au}$ collisions. Conclusions similar to the lower transverse momentum case can be drawn: in particular, close agreement between $R^{0}(q)$ and $R(q)$ indicates absence of any significant angular acceptance issues, decreasing magnitude of correlation moments with increasing order $l$ justifies truncation of the expansion series Eq. (2.1) at $l=4$ and the Gaussian shape assumption leads to a reasonable fit of the correlation moments $\left(\chi^{2} / \mathrm{ndf}=1.6\right)$.

Figure 3 shows the transverse mass, $m_{T}=\sqrt{m^{2}+k_{T}^{2}}$, dependence of the Gaussian radii $\left(R_{\text {out }}=\right.$ $R_{x} / \gamma, R_{\text {side }}=R_{y}$ and $R_{\text {long }}=R_{z}$, where $\gamma$ is the kinematic Lorentz boost in the outward direction from the LCMS frame to the PCMS frame) obtained from the fits to the 3D correlation functions from STAR data (stars). The errors bars on the STAR data are statistical only (systematic uncertainties are negligible compared to statistical uncertainties). Also shown are Gaussian radii for kaon 


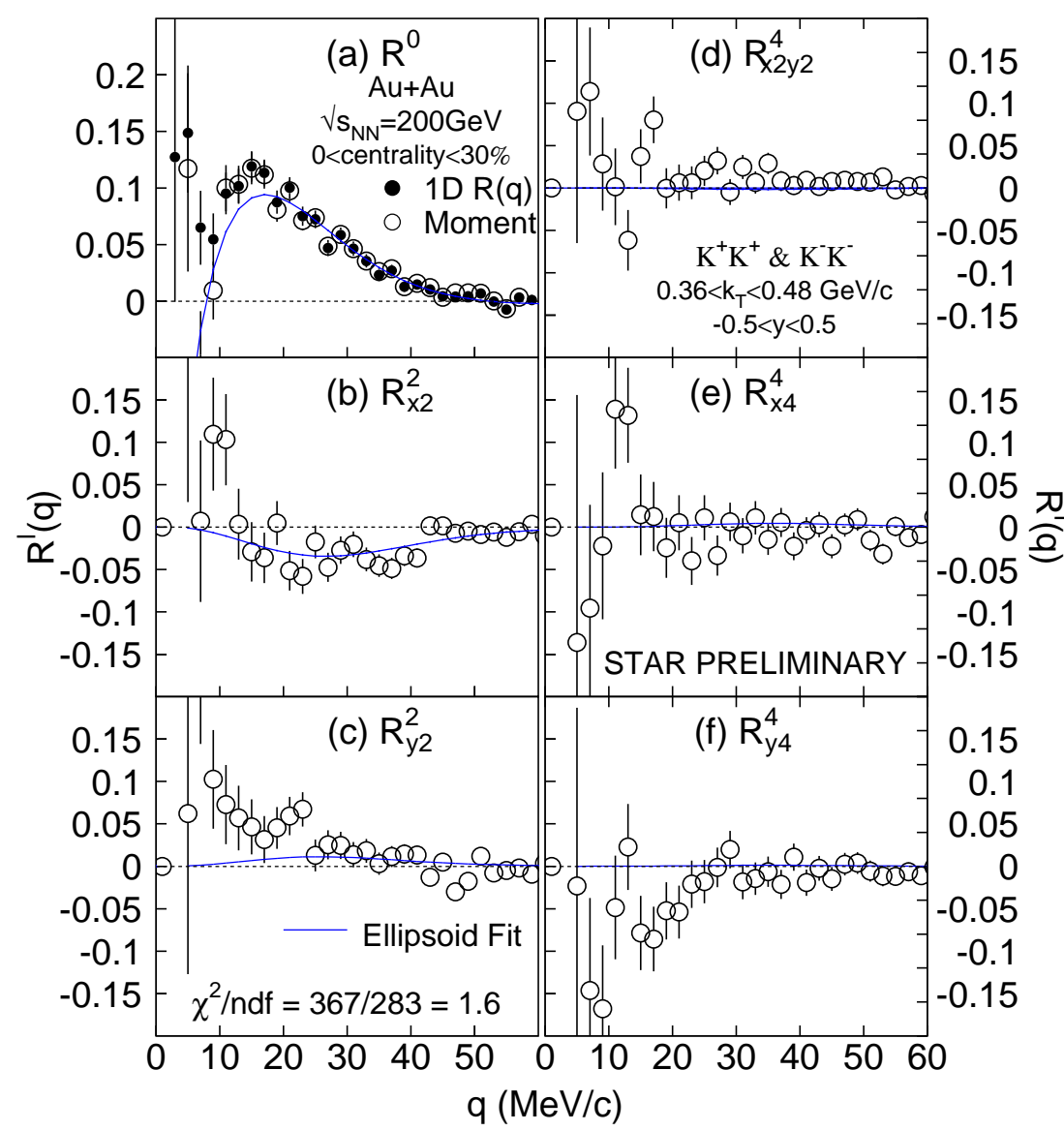

Figure 2: Experimental correlation moments $R^{l}(q)$ for orders $l=0,2,4$ for mid-rapidity kaon pairs $\left(0.36<k_{T}<0.48 \mathrm{GeV} / \mathrm{c}\right)$ from central $(0-30 \%) \mathrm{Au}+$ Au collisions at $\sqrt{s_{N N}}=200 \mathrm{GeV}$. Panel (a) also shows a comparison between $R^{0}(q)$ and $1 D$ correlation function $R(q)$. Systematic errors are negligible compared to statistical errors. The solid curve represents the ellipsoid fit to the data.

data from PHENIX [5] (open circles), with the errors bars representing statistical and systematic uncertainties combined. Model calculations from Buda-Lund model (analytic solution to perfect fluid hydrodynamics) [7] and hydrokinetic model (HKM) (microscopic transport code simulating hydrodynamic expansion of the system followed by dynamic decoupling) [8] are shown in solid curve and solid squares respectively. The dotted lines are to guide the eye only.

\section{Discussions}

From figure 3, it can be observed that the Gaussian radii for the kaon source function display a monotonic decrease with increasing transverse mass $m_{T}$ from the STAR data at low $m_{T}$ to the PHENIX data at higher $m_{T}$, as do the model calculations Buda-Lund and HKM. The Gaussian radii in the outward and sideward directions are adequately described by both models over the $m_{T}$ range up to $1.0 \mathrm{GeV}$. However, there is a marked deviation between the HKM and the Buda-Lund predictions in the longitudinal direction, with the deviation becoming more prominent toward lower $m_{T}$. The increasing agreement between the models at high $m_{T}>0.7 \mathrm{GeV}$ coupled with the large uncertainties on the available data does not lead to a clear conclusion as to the relative validity of 


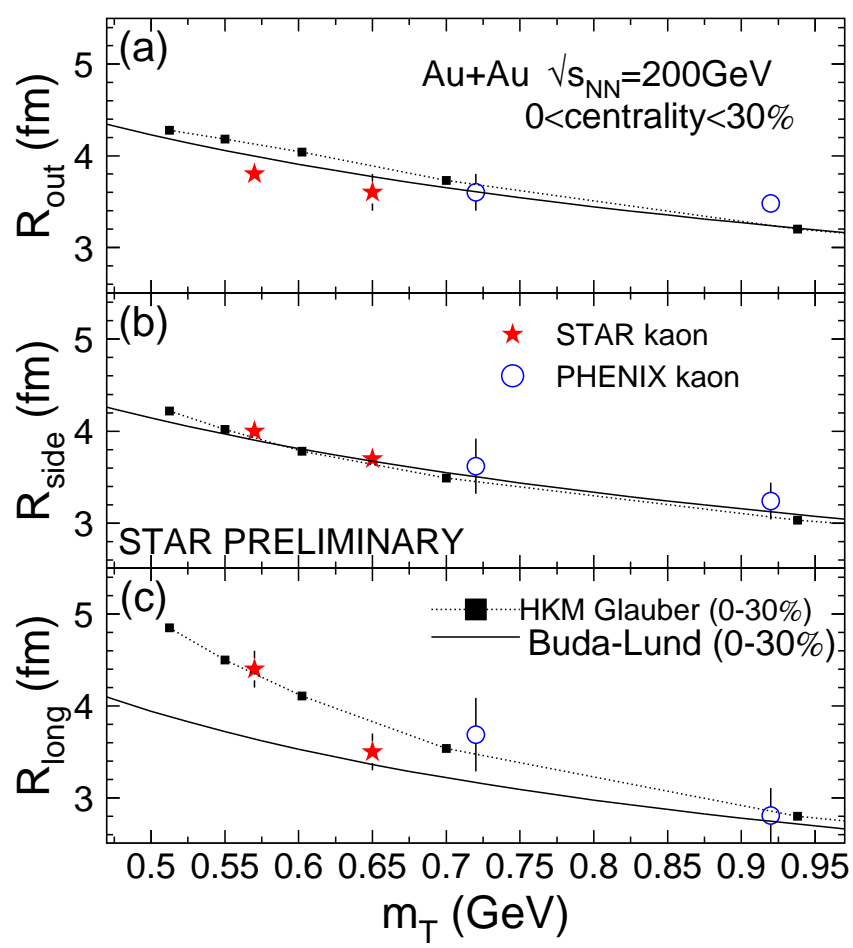

Figure 3: Transverse mass dependence of Gaussian radii (a) $R_{\text {out }},(b) R_{\text {side }}$ and (c) $R_{\text {long }}$ for mid-rapidity kaon pairs from central (0-30\%) Au+Au collisions at $\sqrt{s_{N N}}=200 \mathrm{GeV}$. STAR data are shown in full stars (error bars are statistical only, systematic errors are negligible); PHENIX data in open circles (error bars include both statistical and systematic). Hydrokinetic model and Buda-Lund model calculations are shown by solid squares and solid line respectively.

the two models. On the other hand, the precise STAR measurements at lower $m_{T}$ permits a clear distinction between them: they favor the HKM model as more representative of the expansion dynamics of the fireball. Hence, the $m_{T}$ scaling of the Gaussian radius in the longitudinal direction between kaons and pions, a key feature of the Buda-Lund model, is not supported by these data.

\section{References}

[1] D. A. Brown and P. Danielewicz, Phys. Rev. C 57, 2474 (1998).

[2] M. Lisa et al., Annu. Rev. Nucl. Part. Sci. 55, 357, (2005).

[3] P. Danielewicz and S. Pratt, Phys.Lett.B 61860 (2005).

[4] S. Afanasiev et al. (PHENIX Collaboration), Phys. Rev. Lett. 100, 232301 (2008).

[5] S. Afanasiev et al. (PHENIX Collaboration), Phys. Rev. Lett. 103, 142301 (2009).

[6] P. Chung (STAR Collaboration), Physics of Particles and Nuclei Letters, Vol. 8, No. 9, 1019 (2011).

[7] M. Csanad and T. Csorgo, arXiv:nucl-th/0801.0800v1 (2008).

[8] Iu. A. Karpenko and Yu. M. Sinyukov, Phys. Rev. C81, 054903 (2010).

[9] K. H. Ackermann et al. (STAR Collaboration), Nucl. Instrum. Meth. A. 499 p.624 (2003).

[10] J. Adams et al. (STAR Collaboration), Phys. Rev. C 71, 044906 (2005). 